\title{
Tuning of a PID Controller using Modified Dynamic Group based TLBO Algorithm
}

\author{
Zhiyong Luo \\ School of Advanced \\ Manufacturing \\ Engineering, \\ Chongqing University \\ of Posts and \\ Telecommunications, \\ PR China
}

\author{
Qi Guo \\ School of Automation, \\ Chongqing University \\ of Posts and \\ Telecommunications, \\ PR China
}

\author{
Jie Zhao \\ School of Advanced \\ Manufacturing \\ Engineering, \\ Chongqing University \\ of Posts and \\ Telecommunications, \\ PR China
}

\author{
Shensheng Xu \\ School of Automation, \\ Chongqing University \\ of Posts and \\ Telecommunications, \\ PR China
}

\begin{abstract}
This paper presents a new version of Teaching LearningBased Optimization (TLBO) algorithm to find the optimal parameters of Proportional Integral Derivative (PID) controller. The proposed algorithm is an altered version of dynamic group strategy TLBO (DGS-TLBO) and is named as modified dynamic group based TLBO (MDG-TLBO) algorithm. The proposed algorithm is tested on 12 benchmark functions to verify its efficiency over other procedures. The results show that the MDG-TLBO algorithm offers better solution quality and has better convergence rate. Finally, the proposed algorithm is tested on a three-tank liquid-level control system for the optimization of PID gains. The simulation result indicate that the proposed algorithm is an effective method in tuning of PID controllers to obtain better performance measures the error values and the time domain specifications.
\end{abstract}

\section{General Terms}

Algorithm, PID controller

\section{Keywords}

Teaching-learning-based optimization, Liquid-level controller

\section{INTRODUCTION}

PID controller is a widely used controller in industry. It uses the three parameters known as proportion, integral and deviation to conduct a loop feedback control based on the error value of the system. Because of its so many advantages such as simplicity, ease of use, effectiveness and robustness etc [1,2], more than $90 \%$ of industrial controllers are still implemented based around PID control algorithms [3,4]. In the application of a controller, the setting of the three parameters is the core content of the control system. However, the real problem is that it's difficult to find the precise and optimal PID parameters when using the traditional PID controller optimization methods, such as Ziegler and Nichols (Z-N) method [5], Cohen-Coon method [6]. The results got by traditional method usually should be refined again since they always in an unacceptable performance.

Recently, many optimization methods which based on evolutionary algorithm and swarm intelligence have been employed to tune PID controller parameters. these optimization methods contain such as genetic algorithm (GA) [7], particle swarm optimization (PSO) [8], difference evolution (DE) [9], ant colony optimization (ACO) [10], harmony search (HS) [11]and teaching-learning-based optimization (TLBO) algorithm etc.
The teaching-learning-based optimization algorithm was originally introduced by Rao et al [12]. It's inspired by the teaching-learning process in a classroom, similar to other intelligent algorithms, TLBO is also a population-based algorithm. Due to its simplicity, less deployment parameters and well-performed numerical results, this algorithm has been applied in many engineering fields.

However, in the process of evolutionary computation, the TLBO algorithm hardly avoid being trapped in a local optimal when dealing with some complex problems containing multimodal local optimal solutions, for this reason, a dynamic group strategy was introduced to the original TLBO algorithm. The teaching-learning-based optimization with dynamic group strategy (DGS-TLBO) was proposed by Zou et al [13], it aims at improving the performance of the original TLBO through dynamic group. Tests have demonstrated the DGS-TLBO well-performs than the original TLBO algorithm. But, to some extent, the numerical results displayed in the paper [13] still have the potential to be improved.

In this paper, a modified dynamic group based TLBO (MDGTLBO) is presented. In order to maintain the diversity of population, an improved formula is made to take place of the original one in the teacher phase, then, after the learn phase, a new tutoring phase is proposed to improve the worst student in a group by the group teacher. To validate the performance of MDG-TLBO, tests have been made on the 12 benchmark functions with the same criteria as DGS-TLBO. Then the MDG-TLBO algorithm is used to tune a PID controller.

The remaining of this paper is organized as follows. Section 2 introduces the DGS-TLBO algorithm, the MDG-TLBO algorithm is presented in Section 3. In Section 4, MDG-TLBO is tested on 12 benchmark functions together with the original TLBO and the DGS-TLBO. Section 5 gives a simple application case introduction about three-tank liquid-level system and describes the using of MDG-TLBO to tune the PID controller. Finally, conclusions are given in Section 6.

\section{TEACHING-LEARNING-BASED OPTIMIZATION WITH DYNAMIC GROUP STRATEGY}

Teaching-learning-based optimization (TLBO) is based on the philosophy of teaching and learning and it works on the effect of influence of a teacher on the output of learners in a class [14]. In this algorithm, the population is considered as a group of learners and different design variables related to each learner can be considered as different subjects. Learner's score is analogous to the fitness value of an optimization 
problem and the teacher is considered as the best value in the population.

The process of TLBO is divided into two parts, 'teacher phase' and 'learner phase'. In the teacher phase, all the students learn from the teacher, whereas in the learner phase, students learn through the interaction between other students. More detailed description of original TLBO could refer to the paper [12-18]. In this section, more attention would be focused on the teaching-learning-based optimization with dynamic group strategy (DGS-TLBO).

The DGS-TLBO algorithm has brought some fundamental changes to the original TLBO algorithm by introducing the 'group' concept. It divides the learners into small-sized groups in order to increase the diversity of the population. As all learners belong to certain groups, after a certain number of generations, the learners would be regrouped again, the periodical regrouping can make sure the exchange of information covers all learners so as to improve the exploration ability. The specific process of the algorithm is as follows.

\subsection{Teacher phase}

In this phase, the teacher distributes his knowledge to all students and tries to improve the mean result of the class in the subjects. Let's suppose $X_{\text {best }}$ is the highest learned person who has the best fitness value and is identified and assigned as a teacher. GroupMean is the mean value of the scores obtained by corresponding group of students for each of their subject. After learning from the teacher of class and the mean of his own group, learner $\mathrm{X}$ is updated in the teacher phase according to the following formula:

$$
\begin{aligned}
& \text { newX }=X+r^{*}\left(X_{\text {best }}-T F * \text { GroupMean }\right) \\
& T F=\operatorname{round}[1+\operatorname{rand}(0,1)]
\end{aligned}
$$

where $\mathrm{r}$ is a random number between 0 and $1, \mathrm{TF}$ is the teaching factor which can be set to either 1 or 2 randomly using Eq. 2. newX means the updated value of learner $X$, the new value is accepted only if it gives better function value than the old one.

\subsection{Learner phase}

In DGS-TLBO algorithm, there are two learning modes in the learner phase, the original TLBO learning mode and the quantum-behaved learning mode, each learner can choose either of them by the following pseudo code 1 :

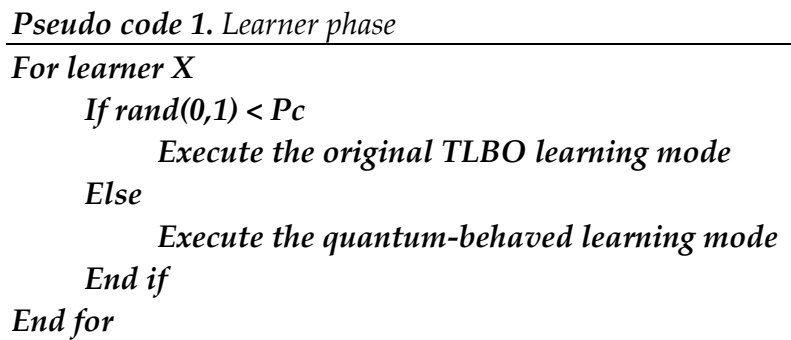

where $\mathrm{Pc}$ is a constant parameter set for learners, in our case, $\mathrm{Pc}$ is set to 0.5 , it has been proved that a large value of Pc would improve the diversity of learners, and a small one would enhance the convergence speed [13].

The original TLBO learning mode can be described using the following pseudo code 2 :
Pseudo code 2. The original TLBO learning mode

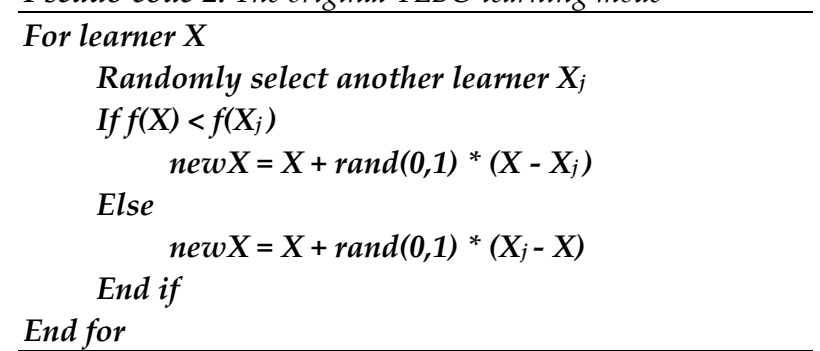

The quantum-behaved learning mode can be described using the following pseudo code 3 :

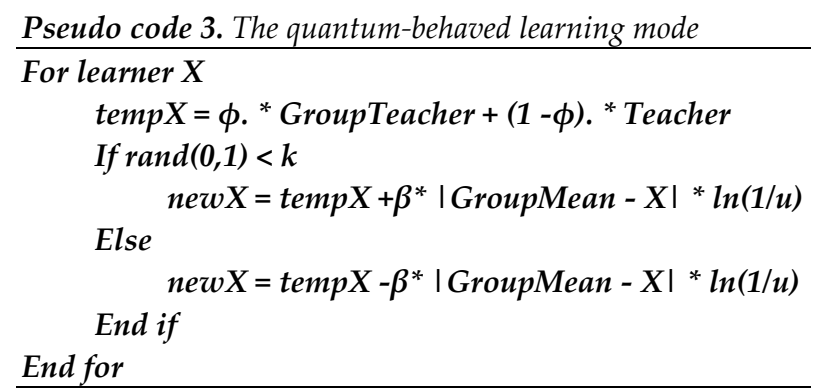

In pseudo code 3, where GroupTeacher means the best learner of learner X's own corresponding group, Teacher and GroupMean have the same explanation as mentioned before. $\Phi, \mathrm{u}, \mathrm{k}$ and $\beta$ are different vectors, in which, each element is a random number between 0 and 1 . After the learning phase, the newX will be accepted if it gives better function value. The Teacher, GroupTeacher, GroupMean will be updated in every generation, after a certain generations, the class will be regrouped again. The algorithm will be ended if the termination criteria is satisfied.

\section{DESCRIPTION OF MODIFIED DYNAMIC GROUP BASED TLBO (MDG-TLBO) \\ 3.1 Modification in teacher phase}

In the DGS-TLBO algorithm, each learner learns from both the Teacher and the GroupMean of his corresponding group, this has a great improvement compared with the original TLBO algorithm. However, it still haven't taken into account that the differences between learners whom in the same group completely. In the proposed MDG-TLBO, a modified parameter GroupMean' is proposed, the formula for updating the value of learner $\mathrm{X}$ in teacher phase is improved as follows:

$$
\begin{aligned}
& \text { GroupMean' }^{\prime}(\text { GroupMean+X }) / 2 \\
& \text { newX }=X+r *(\text { Teacher }-T F * \text { GroupMean' })
\end{aligned}
$$

The advantage of the modification is the parameter GroupMean' differs from learner to learner in each group, that can improve the diversity of the population and therefore enhance the exploration nature of the algorithm further more.

\subsection{Modification after learn phase}

In the MDG-TLBO algorithm, a new phase named tutoring phase is presented and it works after learner phase of each group. The tutoring phase, simulates the one-on-one tutoring between a teacher and a learner, aims to improve the worst learner in the relative group by the teacher of group in a short time. The mathematical formulation of tutoring operation is described as follows: 
$n e w X w=X w+T F *($ GroupTeacher $-X w)$

TF $=2-$ gen/genMax

Here, Xw denotes the worst learner in his group, newXw denotes the updated value of learner Xw, GroupTeacher denotes the best learner in the same group with learner Xw. $\mathrm{TF}$ is the tutoring factor, gen and genMax mean the current generation and the max generation respectively.

The introduction of tutoring phase can have a distinct improvement on the performance of each group through updating the value of the worst learner. Although the new step increase the function evaluations (FEs), compared with the same FEs, the max number of iterations will decrease, however, tests have been made to demonstrate that the reduced number of iterations have little effect on the performance of the algorithm. Fig. 1 illustrates the flowchart of MDG-TLBO algorithm.

\section{TEST FUNCTIONS AND EXPERIMENTAL RESULTS}

In this section, 12 benchmark functions with different characteristics are used to evaluate the performance of the proposed algorithm. The test criteria is as same as that in the DGS-TLBO algorithm. Since other algorithms such as jDE
[19], SaDE [20], PSO-cf-Local [21], FDR-PSO [22] have been compared with DGS-TLBO algorithm, so, here only the original TLBO, DGS-TLBO and MDG-TLBO are made a comparison of their result.

\subsection{Benchmark functions and parameter settings}

The 12 benchmark functions with different traits are described in Table 1. The tests were implemented on an Intel dual-core 3.30GHz CPU, 4 GB RAM, and Windows 7 professional with Matlab R2012a runtime environment. In the experiments, the population size was set to 50 in 10 dimensions for $\mathrm{F} 1$ to $\mathrm{F} 9$, the count of group and regrouping period were set to 5 , the learning ability Pc was set to 0.5 , the termination criteria is a certain number of fitness evaluations about 100000 for 10 dimensions, also 20000 and 40000 for 2 and 4 dimensions respectively. The each function was simulated by various algorithm 50 times independently. The results are shown in Table 2.

\subsection{Experimental results}

The test results are shown in Table 2 and Fig. 2. In Table 2, B, $\mathrm{M}, \mathrm{W}$ and SD denote the best, mean, worst results and the standard deviation respectively. The best results are shown in bold.

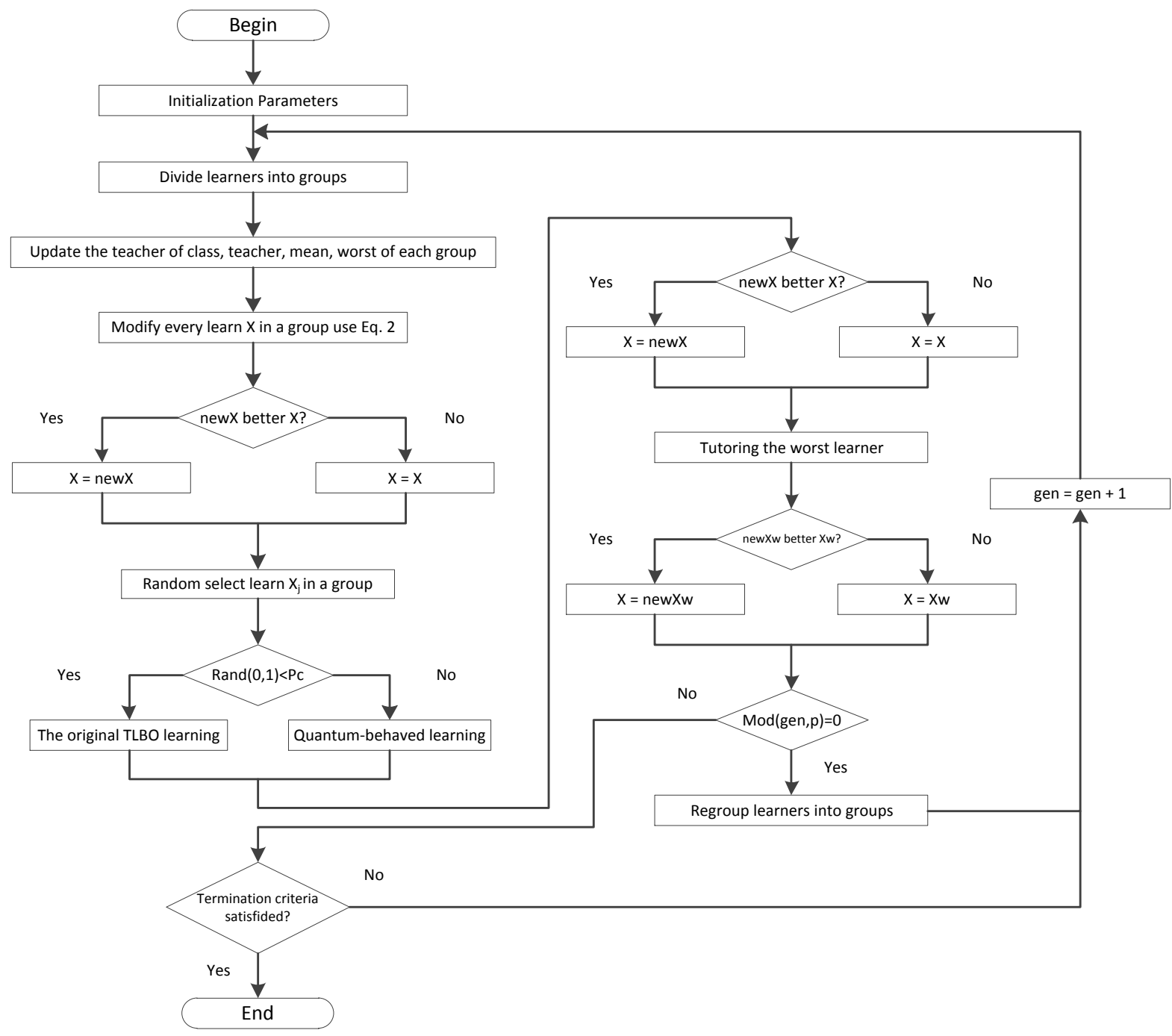

Figure 1. Flowchart of the MDG-TLBO algorithm 
Table 1. Test benchmark functions

\begin{tabular}{lllll}
\hline Function & Formula & Dim. & Range & Optima \\
\hline Sphere & $F_{1}(x)=\sum_{i=1}^{D} x_{i}^{2}$ & 10 & {$[-100,100]$} & 0 \\
Quadric & $F_{2}(x)=\sum_{i=1}^{D}\left(\sum_{j=1}^{i} x_{j}\right)^{2}$ & 10 & {$[-100,100]$} & 0 \\
Sum Square & $F_{3}(x)=\sum_{i=1}^{D} i_{i}^{2}$ & 10 & {$[-10,10]$} & 0 \\
Zakharov & $F_{4}(x)=\sum_{i=1}^{D} x_{i}^{2}+\left(\sum_{i=1}^{D} 0.5 x_{i}\right)^{2}+\left(\sum_{i=1}^{D} 0.5 x_{i}\right)^{4}$ & 10 & {$[-10,10]$} & 0 \\
Rosenbrock & $F_{5}(x)=\sum_{i=1}^{D-1}\left[100\left(x_{i}^{2}-x_{i+1}\right)^{2}+\left(x_{i}-1\right)^{2}\right]$ & 10 & {$[-2.048,2.048]$} & 0 \\
Ackley & $F_{6}(x)=20-20 \exp \left(-\frac{1}{5} \sqrt{\frac{1}{D} \sum_{i=1}^{D} x_{i}^{2}}\right)-\exp \left(\frac{1}{D} \sum_{i=1}^{D} \cos \left(2 \pi x_{i}\right)\right)+\mathrm{e}$ & 10 & {$[-32.768,32.768]$} & 0 \\
Rastrigin & $F_{7}(x)=\sum_{i=1}^{D}\left(x_{i}^{2}-10 \cos \left(2 \pi x_{i}\right)+10\right)$ & 10 & {$[-5.12,5.12]$} & 0 \\
Griewank & $F_{8}(x)=\sum_{i=1}^{D} \frac{x_{i}^{2}}{4000}-\prod_{i=1}^{n} \cos \left(\frac{x_{i}}{\sqrt{i}}\right)+1$ & 10 & {$[-600,600]$} & 0 \\
Schwefel & $F_{9}(x)=418.9829 D+\sum_{i=1}^{D}\left(-x_{i} \sin \sqrt{a b s\left(x_{i}\right)}\right)$ & 10 & {$[-500,500]$} \\
Colville & $F_{10}(x)=100\left(x_{1}^{2}-x_{2}\right)^{2}+\left(x_{1}-1\right)^{2}+\left(x_{3}-1\right)^{2}+90\left(x_{3}^{2}-x_{4}\right)^{2}+$ & & & 0 \\
Matyas & $10.1\left(\left(x_{2}-1\right)^{2}+\left(x_{4}-1\right)^{2}\right)+19.8\left(x_{2}-1\right)\left(x_{4}-1\right)$ & 4 & {$[-10,10]$} \\
Bukin N.6 & $F_{11}(x)=0.26\left(x_{1}^{2}+x_{2}^{2}\right)-0.48 x_{1} x_{2}$ & & & 0 \\
\hline
\end{tabular}

Table 2. Results of 12 benchmark functions over $\mathbf{5 0}$ runs

\begin{tabular}{|c|c|c|c|c|c|c|c|c|c|}
\hline & & TLBO & DGS-TLBO & MDG-TLBO & & & TLBO & DGS-TLBO & MDG-TLBO \\
\hline \multirow{5}{*}{ F1 } & B & $2.5865 \mathrm{e}-206$ & $4.6760 \mathrm{e}-289$ & 0 & \multirow{4}{*}{ F7 } & B & 0 & 0 & 0 \\
\hline & $\mathrm{M}$ & 2.2197e-202 & 6.6737e-283 & 0 & & M & 1.7180 & 0.3399 & 0.3386 \\
\hline & W & 5.4737e-201 & $3.2404 \mathrm{e}-281$ & 0 & & W & 4.9748 & 7.9597 & 5.9698 \\
\hline & SD & 0 & 0 & 0 & & SD & 1.5823 & 1.4156 & 1.3586 \\
\hline & B & $1.2957 \mathrm{e}-95$ & $1.6863 \mathrm{e}-156$ & 0 & \multirow{4}{*}{ F8 } & B & 0 & 0 & 0 \\
\hline \multirow{4}{*}{$\mathrm{F} 2$} & $\mathrm{M}$ & $6.7700 \mathrm{e}-91$ & $1.7046 \mathrm{e}-148$ & $9.6274 \mathrm{e}-320$ & & M & 0.0038 & 0.0127 & 0.0143 \\
\hline & W & $2.1939 \mathrm{e}-89$ & $6.0299 \mathrm{e}-147$ & $3.3303 e-318$ & & W & 0.0590 & 0.0836 & 0.1229 \\
\hline & SD & $3.1548 \mathrm{e}-90$ & $8.7322 \mathrm{e}-148$ & 0 & & SD & 0.0099 & 0.0228 & 0.0326 \\
\hline & B & $8.8131 \mathrm{e}-208$ & $1.0925 \mathrm{e}-289$ & 0 & \multirow{4}{*}{ F9 } & B & 118.4385 & 236.8768 & 335.5781 \\
\hline \multirow{3}{*}{ F3 } & $\mathrm{M}$ & $2.3646 \mathrm{e}-203$ & $8.7255 \mathrm{e}-286$ & 0 & & M & 520.2401 & 617.6238 & 760.1029 \\
\hline & $\mathrm{W}$ & 5.4896e-202 & $1.4023 \mathrm{e}-284$ & 0 & & $\mathrm{~W}$ & $1.0462 \mathrm{e}+03$ & $1.0280 \mathrm{e}+03$ & $1.2436 \mathrm{e}+03$ \\
\hline & SD & 0 & 0 & 0 & & SD & 202.0300 & 201.0992 & 216.1216 \\
\hline \multirow{4}{*}{$\mathrm{F} 4$} & B & $4.7589 \mathrm{e}-105$ & $1.1099 \mathrm{e}-161$ & 0 & \multirow{4}{*}{ F10 } & B & $6.0601 \mathrm{e}-08$ & $1.5802 \mathrm{e}-15$ & 0 \\
\hline & $\mathrm{M}$ & $7.7242 \mathrm{e}-100$ & $1.8790 \mathrm{e}-156$ & 0 & & $\mathrm{M}$ & $2.8394 \mathrm{e}-04$ & 0.0886 & $1.1839 \mathrm{e}-28$ \\
\hline & $\mathrm{W}$ & 2.0133e-98 & 6.4456e-155 & $1.4822 \mathrm{e}-323$ & & $\mathrm{~W}$ & 0.0076 & 3.8195 & $2.7895 \mathrm{e}-27$ \\
\hline & SD & 2.8571e-99 & $9.1680 \mathrm{e}-156$ & 0 & & SD & 0.0011 & 0.5403 & $4.8085 \mathrm{e}-28$ \\
\hline \multirow{4}{*}{ F5 } & B & $4.2259 \mathrm{e}-05$ & 2.9264 & $2.7748 \mathrm{e}-18$ & \multirow{4}{*}{ F11 } & B & $2.5764 \mathrm{e}-58$ & $3.9382 e-95$ & $2.9184 \mathrm{e}-142$ \\
\hline & $\mathrm{M}$ & 0.0048 & 4.6524 & $5.6819 \mathrm{e}-05$ & & $\mathrm{M}$ & $6.8526 \mathrm{e}-53$ & $2.3475 e-87$ & $2.1540 \mathrm{e}-136$ \\
\hline & $\mathrm{W}$ & 0.0965 & 6.0931 & 0.0025 & & $\mathrm{~W}$ & $2.7387 \mathrm{e}-51$ & $9.7273 e-86$ & $6.1610 \mathrm{e}-135$ \\
\hline & SD & 0.0158 & 0.4428 & $3.5797 \mathrm{e}-04$ & & SD & $3.8736 \mathrm{e}-52$ & $1.3782 \mathrm{e}-86$ & $1.0207 \mathrm{e}-135$ \\
\hline \multirow{4}{*}{ F6 } & B & 0 & 0 & 0 & \multirow{4}{*}{ F12 } & B & 0.0500 & 0.0328 & 0.0037 \\
\hline & $\mathrm{M}$ & $3.0553 e-15$ & $3.1264 \mathrm{e}-15$ & $2.0606 \mathrm{e}-15$ & & $\mathrm{M}$ & 0.0671 & 0.0491 & 0.0455 \\
\hline & W & $3.5527 \mathrm{e}-15$ & $3.5527 \mathrm{e}-15$ & $3.5527 \mathrm{e}-15$ & & $\mathrm{~W}$ & 0.3493 & 0.0507 & 0.0500 \\
\hline & SD & $1.2453 \mathrm{e}-15$ & $1.1662 e-15$ & $1.7713 \mathrm{e}-15$ & & SD & 0.0450 & 0.0037 & 0.0101 \\
\hline
\end{tabular}




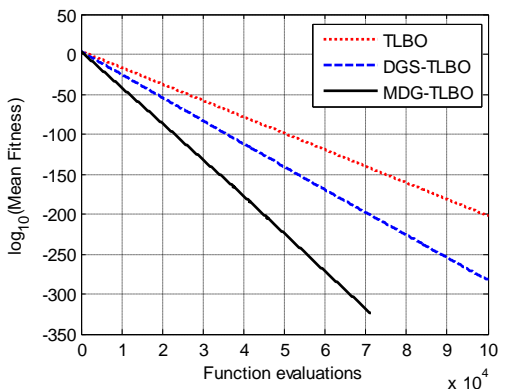

(F1) Sphere

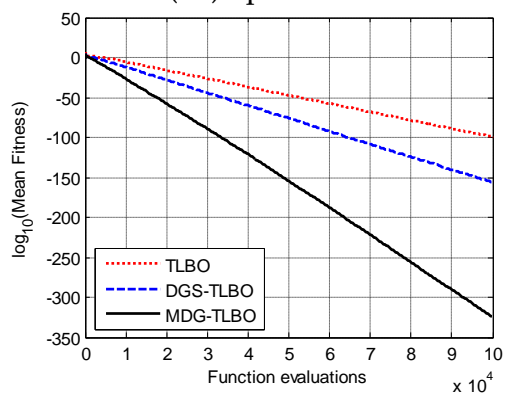

(F4) Zakharov

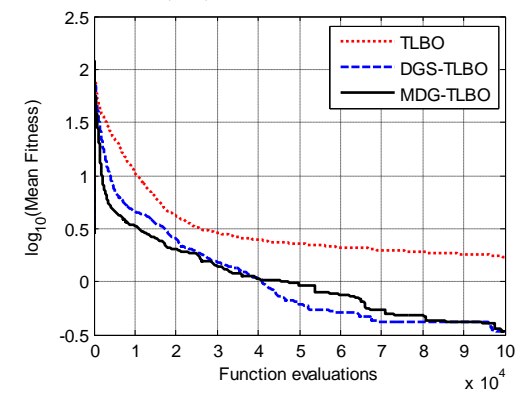

(F7) Rastrigin

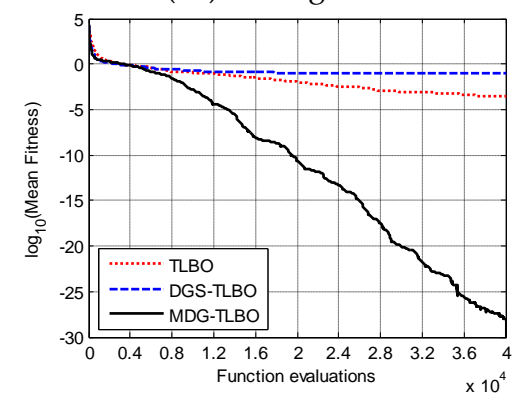

(F10) Colville

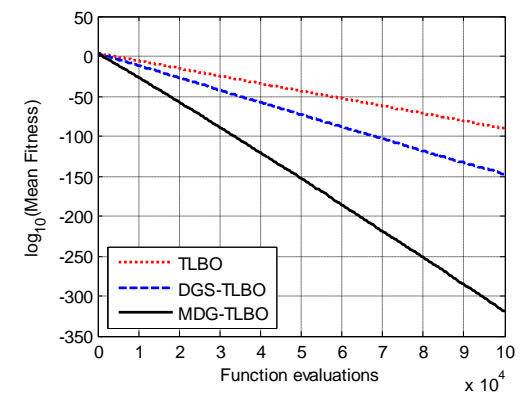

(F2) Quadric

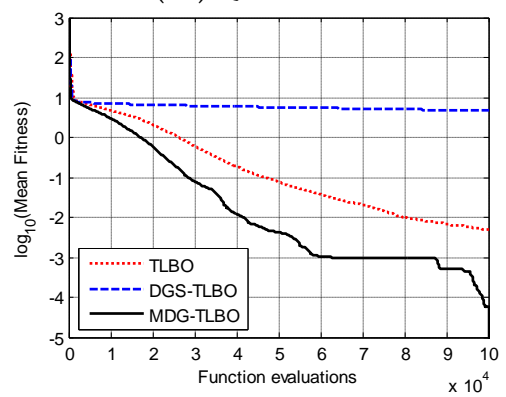

(F5) Rosenbrock

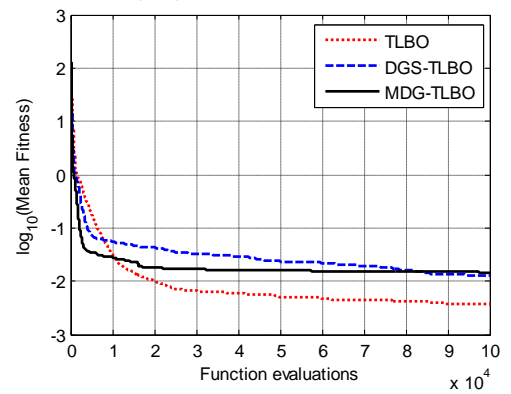

(F8) Griewank

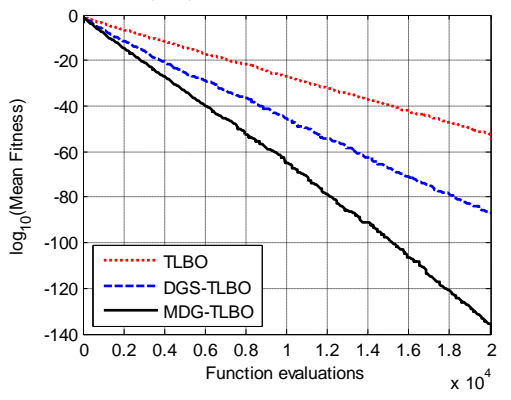

(F11) Matyas

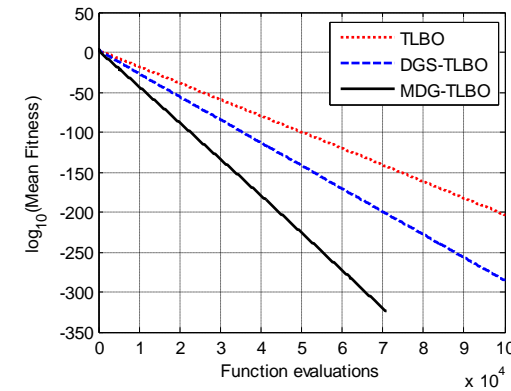

(F3) Sum Square

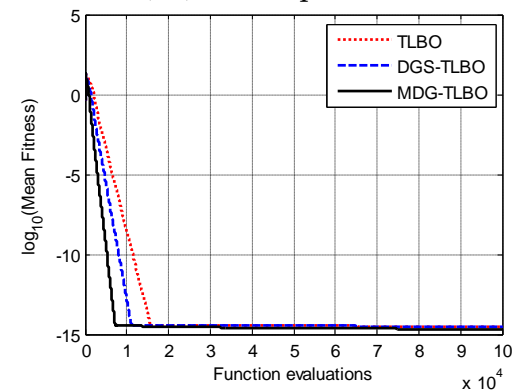

(F6) Ackley

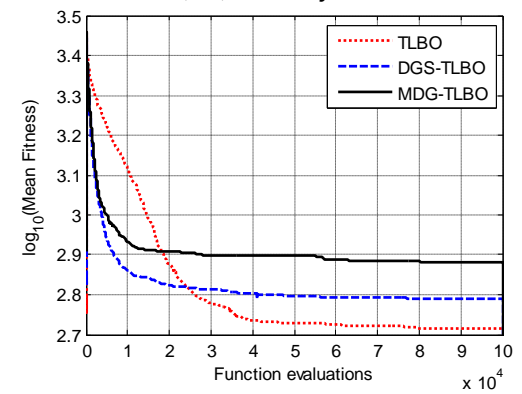

(F9) Schwefel

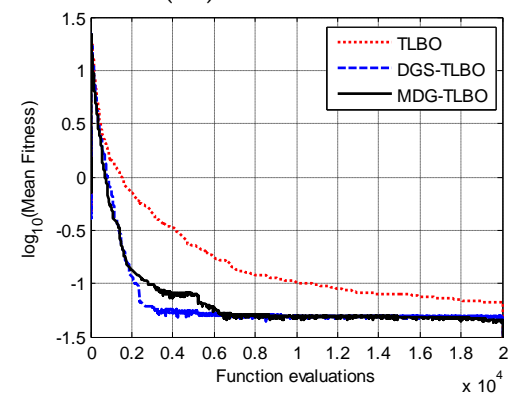

(F12) Bukin N.6

Figure 2. Convergence of the 3 algorithms on test functions.

From Table 2, it can been observed that MDG-TLBO algorithm performs well in terms of all considered metrics for function F1, F2, F3, F4, F5, F10, and F11. For function F6, F7 and F8, all three algorithms can obtain the best solution, especially, the original TLBO algorithm performs well for function F8. For function F6, F9 and F12, the DGS-TLBO algorithm has a better performance in standard deviation. For function 12, the MDG-TLBO performs well except in standard deviation.

The convergence rate of all three algorithms for 12 benchmark functions have been graphically presented in Fig. 2. It can be seen clearly from Fig. 2 that the proposed MDGTLBO algorithm has better convergence characteristic for most functions compared with the original TLBO and DGSTLBO algorithms. Therefore, it can be concluded that the
MDG-TLBO performs well in terms of getting better solution accuracy together with convergence speed.

\section{TUNING OF A PID CONTROLLER}

After having validated the performance of MDG-TLBO on the 12 benchmark functions, a classic case about three-tank liquid-level system will be studied, and the MDG-TLBO is used to tune the PID controller. Three-tank liquid-level instrument is a typical nonlinear time-delay process control system, it's very important in many industrial applications such as chemical industry and water purification systems [3].

The simple structure of three-tank liquid-level system is shown in Fig. 3(a). The main principle of the system can be described as follows: the water in the tank $\mathrm{A}$ is piped to tank $\mathrm{B}, \mathrm{C}$ and $\mathrm{D}$ by a pump, water level of each tank is measured by sensors and the difference value between actual level and 
set point is calculated, then the PID controller response feedback to regular the flow-rate based on the error value of the system

More details about three-tank liquid-level could refer to the paper $[23,24]$. In this section, more attention would be paid on the tuning of the PID controller with MDG-TLBO algorithm, the overall transfer function of the system is given by [3] as:

$$
G(s)=\frac{1}{64 s^{3}+9.6 s^{2}+0.48 s+0.008}
$$

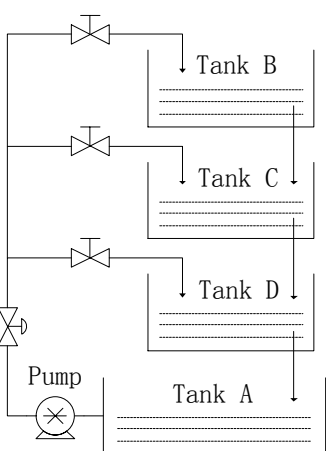

(a)
The block diagram of the three-tank liquid-level system with a PID controller is shown in Fig. 3(b). Tuning of the PID controller with MDG-TLBO algorithm focus on obtaining the optimal solution for the three PID gains $\mathrm{Kp}, \mathrm{Ki}$, and $\mathrm{Kd}$ by minimizing the objection function [25]. Here, the minimization of the integral square error (ISE) has been carried out. In this section, other algorithms such as GA, PSO, and Ziegler-Nichols tuning method will be compared with the proposed algorithm.

Figure 3. The Simple structure and block diagram of the three-tank liquid level system. (a)Simple structure of the system. (b)Block diagram of closed loop system controlled by PID

$$
J_{I S E}=\int_{0}^{\infty}[r(t)-h(t)]^{2} d t=\int_{0}^{\infty} e^{2}(t) d t
$$

For all the algorithms, the upper and lower bounds of PID parameters are considered as: $\mathrm{Kp}:\left[\begin{array}{ll}0 & 1\end{array}\right], \mathrm{Ki}:\left[\begin{array}{ll}0 & 1\end{array}\right], \mathrm{Kd}:\left[\begin{array}{ll}0 & 1\end{array}\right]$, the population size is set to 20 , the max function evaluations is set to 1000. For GA, the cross rate $\mathrm{CR}=0.6$. For PSO, the inertia weight $\omega=0.6$, acceleration constant $\mathrm{C} 1$ and $\mathrm{C} 2=2$.

Table 3. Comparison of the experimental results

\begin{tabular}{lllll}
\hline & $\mathrm{K}_{\mathrm{p}}$ & $\mathrm{K}_{\mathrm{i}}$ & $\mathrm{K}_{\mathrm{d}}$ & $\mathrm{ISE}$ \\
\hline Z-N & 0.0567 & 0.0009 & 0.2840 & 22.9442 \\
GA & 0.0468 & 0.0011 & 0.8916 & 8.1242 \\
PSO & 0.0965 & 0.0003 & 1 & 10.0624 \\
TLBOs & 0.0419 & 0.0009 & 1 & $\mathbf{7 . 7 5 0 3}$ \\
\hline & $\mathrm{M}_{\mathrm{p}}$ & $\mathrm{T}_{\mathrm{r}}$ & $\mathrm{T}_{\mathrm{s}}$ & $\mathrm{T}_{\mathrm{p}}$ \\
\hline Z-N & 0.5526 & 25.0052 & 295.7311 & 40.3608 \\
GA & 0.1879 & 20.6592 & $\mathbf{4 1 . 9 7 9 0}$ & 30.1590 \\
PSO & 0.3767 & $\mathbf{1 6 . 6 3 6 9}$ & 154.7817 & $\mathbf{2 5 . 7 5 6 7}$ \\
TLBOs & $\mathbf{0 . 1 2 5 0}$ & 20.7360 & 59.0159 & 27.2240 \\
\hline
\end{tabular}

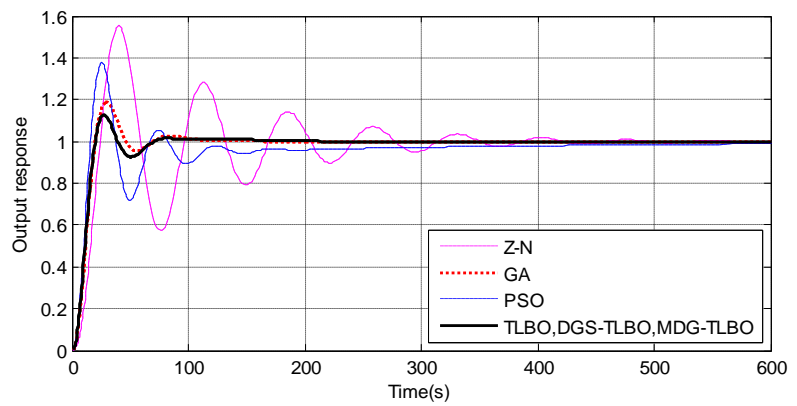

Figure 4. Comparative step response of the system with various PID controllers
The mean results of the step response characteristic and convergence performance of the three-tank liquid-level system for 20 independently runs are shown in Table 3, Fig. 4 and Fig. 5. In Table 3, $\mathrm{M}_{\mathrm{p}}, \mathrm{T}_{\mathrm{r}}, \mathrm{T}_{\mathrm{s}}$ and $\mathrm{T}_{\mathrm{p}}$ denote overshoot, rise time, setting time and peak time respectively, TLBOs means the original TLBO, DGS-TLBO and MDG-TLBO algorithms, which have the same experimental results.

From Table 3 and Fig. 4 it can be observed that all the 3 TLBO-based algorithms can get better result than other compared algorithms based on the ISE criteria. From Fig. 5 it can be observed that the proposed algorithm have a better convergence rate among the 3 algorithms. Therefore, it could be concluded that the algorithm this paper proposed is not only suitable for the application of tuning of a PID controller but also shows excellent performance.

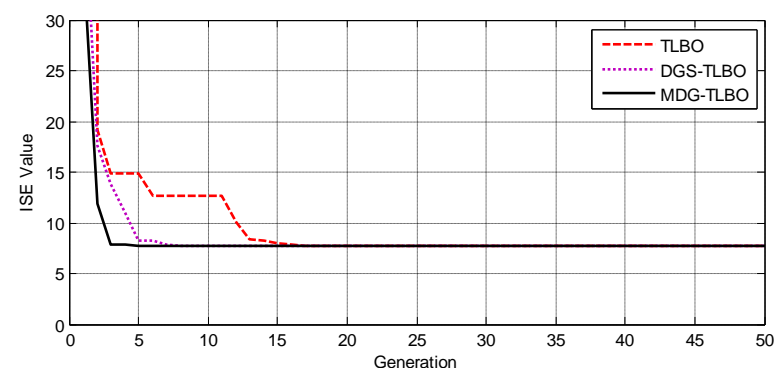

Figure 5. Convergence performance of the 3 TLBO-PID controllers

\section{CONCLUSION}

In this paper, a modified dynamic group based TLBO (MDGTLBO) algorithm is proposed and used to tune a PID controller of a three-tank liquid-level system for obtaining the optimal parameter values. The proposed algorithm has been tested on a set of 12 benchmark functions to validate its performance. The experimental results indicate that the MDGTLBO has significant improvement and better performance compared with original algorithms. Then the new algorithm is 
used to tune a PID controller and simulation results show that the proposed algorithm can obtain the optimal parameter values of PID controller efficiently. Further work is mainly focused on modifying the proposed algorithm suitably for multi-objective optimization problems and using it to solve optimization problems in the other practical applications.

\section{REFERENCES}

[1] Yaghoobi, S.; Mojallali, H. Tuning of a pid controller using improved chaotic krill herd algorithm. Optik 2016, $127,4803-4807$

[2] Priyambada, S.; Mohanty, P.K.; Sahu, B.K. In Automatic voltage regulator using tlbo algorithm optimized pid controller, 9th IEEE International Conference on Industrial and Information Systems, ICIIS 2014, December 15, 2014 - December 17, 2014, Gwalior, India, 2015; Institute of Electrical and Electronics Engineers Inc.: Gwalior, India.

[3] Moharam, A.; El-Hosseini, M.A.; Ali, H.A. Design of optimal pid controller using hybrid differential evolution and particle swarm optimization with an aging leader and challengers. Applied Soft Computing 2016, 38, 727-737.

[4] Iruthayarajan, M.W.; Baskar, S. Evolutionary algorithms based design of multivariable pid controller. Expert Systems with Applications 2009, 36, 9159-9167.

[5] Ziegler, J.G.; Nichols, N.B. Optimum settings for automatic controllers. InTech 1995, 42, 94-100.

[6] Cohen, G.; Coon, G. Theoretical consideration of retarded control. Trans. 1953, 75, 827-834.

[7] Jaen-Cuellar, A.Y.; Romero-Troncoso, R.D.J.; MoralesVelazquez, L.; Osornio-Rios, R.A. Pid-controller tuning optimization with genetic algorithms in servo systems. International Journal of Advanced Robotic Systems 2013, 10 .

[8] Gaing, Z.-L. A particle swarm optimization approach for optimum design of pid controller in avr system. IEEE Transactions on Energy Conversion 2004, 19, 384-391.

[9] Storn, R.; Price, K. Differential evolution - a simple and efficient heuristic for global optimization over continuous spaces. Journal of Global Optimization 1997, $11,341-359$

[10] Hanifah, R.A.; Toha, S.F.; Ahmad, S. In Pid-ant colony optimization (aco) control for electric power assist steering system for electric vehicle, Smart Instrumentation, Measurement and Applications (ICSIMA), 2013 IEEE International Conference on, 2527 Nov. 2013, 2013; pp 1-5.

[11] Wang, H.; Yuan, X.; Wang, Y.; Yang, Y. Harmony search algorithm-based fuzzy-pid controller for electronic throttle valve. Neural Computing and Applications 2013, 22, 329-336.

[12] Rao, R.V.; Savsani, V.J.; Vakharia, D.P. Teachinglearning-based optimization: A novel method for constrained mechanical design optimization problems. Computer-Aided Design 2011, 43, 303-315.

[13] Zou, F.; Wang, L.; Hei, X.H.; Chen, D.B.; Yang, D.D. Teaching-learning-based optimization with dynamic group strategy for global optimization. Information Sciences 2014, 273, 112-131.
[14] Rao, R.V.; Patel, V. An elitist teaching-learning-based optimization algorithm for solving complex constrained optimization problems. International Journal of Industrial Engineering Computations 2012.

[15] Rao, R.V.; Savsani, V.J.; Vakharia, D.P. Teachinglearning-based optimization: An optimization method for continuous non-linear large scale problems. Information Sciences 2012, 183, 1-15.

[16] Sahu, B.K.; Pati, S.; Mohanty, P.K.; Panda, S. Teachinglearning based optimization algorithm based fuzzy-pid controller for automatic generation control of multi-area power system. Applied Soft Computing 2015, 27, 240249.

[17] Suresh C Satapathy, A.N. A modified teaching-learningbased optimization (mtlbo) for global search. Recent Patents on Computer Science 2013, 6, 60-72.

[18] V. Rajinikanth, S.C.S. Design of controller for automatic voltage regulator using teaching learning based optimization. Procedia Technology 2015, vol.21, 295302.

[19] Brest, J.; Greiner, S.; Bokovic, B.; Mernik, M.; Zumer, V. Self-adapting control parameters in differential evolution: A comparative study on numerical benchmark problems. IEEE Transactions on Evolutionary Computation 2006, 10, 646-657.

[20] Qin, A.K.; Huang, V.L.; Suganthan, P.N. Differential evolution algorithm with strategy adaptation for global numerical optimization. IEEE Transactions on Evolutionary Computation 2009, 13, 398-417.

[21] Kennedy, J.; Mendes, R. In Population structure and particle swarm performance, 2002 Congress on Evolutionary Computation, CEC 2002, May 12, 2002 May 17, 2002, Honolulu, HI, United states, 2002; IEEE Computer Society: Honolulu, HI, United states, pp 16711676.

[22] Peram, T.; Veeramachaneni, K.; Mohan, C.K. In Fitnessdistance-ratio based particle swarm optimization, 2003 IEEE Swarm Intelligence Symposium, SIS 2003, April 24, 2003 - April 26, 2003, Indianapolis, IN, United states, 2013; Institute of Electrical and Electronics Engineers Inc.: Indianapolis, IN, United states, pp 174181

[23] Mancilla, M.M.A.; Ramirez, Z.S.; Marin, J.A. In Liquid level nonlinear control for a serial coupled tank system, 2014 IEEE International Autumn Meeting on Power, Electronics and Computing, ROPEC 2014, November 5, 2014 - November 7, 2014, Ixtapa, Mexico, 2014; Institute of Electrical and Electronics Engineers Inc.: Ixtapa, Mexico.

[24] Cartes, D.; Wu, L. Experimental evaluation of adaptive three-tank level control. ISA Transactions 2005, 44, 283 293.

[25] Singh, S.K.; Katal, N.; Modani, S.G. Multi-objective optimization of pid controller for coupled-tank liquidlevel control system using genetic algorithm. In Proceedings of the second international conference on soft computing for problem solving, Babu, B.V.; Nagar, A.; Deep, K.; Pant, M.; Bansal, J.C.; Ray, K.; Gupta, U., Eds. 2014; Vol. 236, pp 59-66. 